Témoigner Témoigner. Entre histoire et mémoire

Getuigen Revue pluridisciplinaire de la Fondation Auschwitz

122 | 2016

Révisionisme et négationisme

\title{
La pédagogie pour contrer le négationnisme
}

Education as a weapon against negationism

Het onderwijs als wapen tegen het negationisme

\section{Baudouin Massart}

\section{(2) OpenEdition}

Journals

Édition électronique

URL : https://journals.openedition.org/temoigner/4152

DOI : $10.4000 /$ temoigner.4152

ISSN : 2506-6390

Éditeur :

Éditions du Centre d'études et de documentation Mémoire d'Auschwitz, Éditions Kimé

Édition imprimée

Date de publication : 2 mai 2016

Pagination : 72-80

ISSN : 2031-4183

Référence électronique

Baudouin Massart, «La pédagogie pour contrer le négationnisme », Témoigner. Entre histoire et mémoire [En ligne], 122 | 2016, mis en ligne le 30 septembre 2021, consulté le 06 janvier 2022. URL http://journals.openedition.org/temoigner/4152; DOI : https://doi.org/10.4000/temoigner.4152 


\section{La pédagogie pour contrer le négationnisme}

$\rightarrow$ Baudouin Massart

Mémoire d'Auschwitz ASBL
(1) La Cellule Démocratie ou barbarie coordonne les questions d'enseignement à la citoyenneté et aux droits humains à travers le travail d'histoire et l'éducation à la mémoire : http://www. democratieoubarbarie.cfwb.be

(2) Des textes et des outils sont repris sur le site http://www. democratieoubarbarie.cfwb.be/ index.php?id=15169

(3) Pierre Vidal-Naquet, Les Assassins de la mémoire, Paris, Maspéro, [1981] ; rééd. Paris, La Découverte, 1987, 238 p.

(4) https://www.kazernedossin.eu

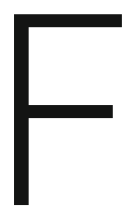

ormer des esprits critiques reste l'une des meilleures façons de lutter contre le négationnisme. Les enseignants - en particulier les professeurs d'histoire de l'enseignement secondaire - sont en première ligne pour mener à bien cette mission. Face aux « J’y crois pas», ils doivent montrer que l'Histoire n'est pas une affaire de croyances, mais une démarche scientifique basée sur des faits réels et vérifiés. Une tâche pas toujours aisée. Pour les épauler, la Cellule Démocratie ou barbarie ${ }^{1}$ de la Fédération Wallonie-Bruxelles a organisé le 25 janvier 2016 une «journée d’étude consacrée aux génocides face au négationnisme et au révisionnisme. $»^{2}$

«Le génocide est l’un des pires crimes qui puisse exister », rappelle le directeur de Démocratie ou barbarie, Olivier Plasman. Et le négationnisme fait partie intégrante du processus, précise-t-il : «Une fois le forfait accompli, les génocidaires prennent bien soin d'effacer toutes les traces.» À travers l'analyse des génocides juif, arménien et tutsi, les participants de cette journée mettent en avant les faits face aux discours de ceux que Pierre Vidal-Naquet appelait «Les Assassins de la mémoire » ${ }^{3}$. De nombreux enseignants et Centres de ressources ont pu aussi confronter leurs expériences et leurs solutions.

\section{NÉGATIONNISME ET RÉVISIONNISME}

Pour entamer le débat, une mise au point s’impose : la distinction entre révisionnisme et négationnisme. «Quand on parle de révisionnisme, explique Laurence Schram, docteure en Histoire, chercheuse senior au Mémorial et Musée Kaserne $\operatorname{Dossin}^{4}$, il s'agit d'une démarche scientifique critique qui vise à réviser de manière rationnelle certaines opinions couramment admises en histoire par le grand public ou par des non-spécialistes. Il se fonde sur des sources nouvelles et propose une nouvelle interprétation de l'histoire, le tout en satisfaisant aux règles de la critique historique.» À l'opposé, elle place le négationnisme qui, lui, ne relève en aucun cas d'une démarche scientifique : c'est « un mensonge qui ne se fonde ni sur une démarche critique ni sur une analyse innovante des sources. Il s'agit d'une démarche perverse et malveillante. L'objectif est de disculper l'Allemagne, de rendre les Juifs coupables et responsables de la Seconde Guerre mondiale et de semer le doute dans 
les esprits en brodant autour de la vérité.» Une rhétorique déjà présente dans les discours d'Adolf Hitler et reprise par le négationniste Robert Faurisson, qui affirme que les chambres à gaz et le génocide ne sont que des mensonges, dont les principales victimes sont les Allemands et les Palestiniens.

\section{LE PROCESSUS DE NÉGATION DE LA SHOAH}

Pour Laurence Schram, l'histoire du négationnisme se découpe en cinq périodes. Elle naît parallèlement à la création de l'État d'Israël, entre 1948 et 1967. Maurice Bardèche, militant d'extrême-droite, publie Nuremberg ou la Terre promise en $1948^{5}$. Il accuse les Alliés d'avoir inventéle génocide pour passer sous silence leurs propres crimes de guerre. En 1950, Paul Rassinier, militant d'extrême gauche, pacifiste, ancien résistant et déporté à Ravensbrück et Dora, conteste, dans Le mensonge d'Ulysse, les témoignages des autres déportés, parce qu'ils ne correspondent pas à son expérience. Il affirme que les principaux bourreaux étaient les kapos et non les SS. Plus tard, dans Ulysse trahi par les siens, il affirme que les Juifs sont la cause de tout. Rassinier est souvent considéré comme le père du négationnisme.

L’année 1967 marque une rupture. «La guerre des Six Jours montre un état juif agressif et guerrier, loin de l'image des victimes », explique Laurence Schram. Rassinier meurt la même année. François Duprat, ancien collaborateur de Bardèche et cadre du FN, prend le relais. Il structure le discours négationniste et convertit l'extrême droite à l'antisionisme. Ce ne sont plus seulement les Allemands les victimes, mais aussi les Arabes. Le négationnisme entre dans une phase de maturation qui va durer jusqu'en 1978. En Belgique, la maison d'édition Baucens diffuse des écrits négationnistes, tandis qu'en Flandre, de futurs dirigeants du Vlaams Blok fondent la revue Haro. En Allemagne, un ancien SS du camp d'Auschwitz, publie en 1973 une brochure Die Auschwitz-Lüge : il y fait une description idyllique d'Auschwitz. En 1978, toujours en Allemagne, Udo Walendy, politologue et activiste d'extrême droite, prétend que les photos des camps sont truquées. La même année, Richard Harwood de son vrai nom, Richard Verrall, un activiste du National Front anglais - publie Did Six Million Really Die ? The Truth at Last, où il nie l'extermination des Juifs. Il se fait passer pour un spécialiste de la Seconde Guerre mondiale de l'Université de Londres, où il est totalement inconnu.

Ces éléments favorisent la propagation du négationnisme en 1978-1985. «C'est l'ère de Robert Faurisson et de la médiatisation et du scandale», souligne Laurence Schram. Le 28 octobre 1978, L’Express publie l'interview «À Auschwitz, on n'a gazé que les poux », de Darquier de Pellepoix, ancien commissaire général aux questions juives dans le gouvernement de Vichy et réfugié en Espagne. L'article est relayé par Le Monde, France Inter... Professeur de littérature à l'Université de Lyon II, Robert Faurisson s'engouffre dans la brèche. Il joue sur sa notoriété et se rallie une frange de l'ultra-gauche, pour qui Auschwitz est une invention du grand capital pour détourner
(5) Le livre est traduit en néerlandais en 1952, par Karel Dillen, futur président du Vlaams Blok. 
La pédagogie pour contrer le négationnisme (suite)

(6) Loi du 23 mars 1995 tendant à réprimer la négation, la minimisation, la justification ou l'approbation du génocide commis par le régime nationalsocialiste allemand pendant la Seconde Guerre mondiale. l'attention de la masse ouvrière de la révolution. En 1979, l'Institute for Historical Review voit le jour aux États-Unis : il devient rapidement un centre international du négationnisme. En Grande-Bretagne, David Irving s'attèle à démontrer qu'Hitler ignorait tout, minimise l'action criminelle du nazisme, ainsi que l'impossibilité technique des chambres à gaz... En Belgique, ces discours sont traduits et relayés, à partir de 1984, par le groupe VHO, porté par l'extrême droite flamande.

De 1985 à 2000, c'est le temps des affaires. L'historien Henri Rousso invente le terme «négationnisme», qui ne se limite pas à nier la Shoah, mais tous les grands crimes de masse du $20^{\mathrm{e}}$ siècle. Faurisson surfe sur les scandales qui ne font quaccroître son audience, pointe Laurence Schram. Il y a aussi l'affaire de la thèse de Roque sur les confessions de Kurt Gerstein à l'université de Nantes. Ou encore l'affaire Garaudy sur Les Mythes fondateurs de la politique israélienne (1995), dans laquelle se compromet l'Abbé Pierre. C'est aussi l'époque des jeux de mots de JeanMarie Le Pen (ex. : «Durafour crématoire»). Derrière ces affaires, plane l'ombre de Faurisson. Une nouvelle génération de négationnistes se profile. Ils sont plus radicaux que leurs prédécesseurs. Pour les contrer, la France adopte la loi Gayssot en 1990 qui condamne la contestation de l'existence des crimes contre l'humanité. La Belgique lui emboîte le pas en $1995^{6}$.

Depuis 2000, le négationnisme diffuse largement ses thèses via Internet et les réseaux sociaux. «Il sort de la culture underground et des groupuscules néonazis. Très médiatisé dans certains pays (pays arabes, ex-républiques soviétiques, Venezuela, Turquie, Soudan...), il connaît une nouvelle évolution », observe Laurence Schram. En 2006, l'Iran organise un colloque négationniste auquel assistent Dieudonné et Faurisson. Thèse avancée : si Israël tire sa légitimité de la Shoah, et si la Shoah n’a pas eu lieu, alors Israël n’a pas de légitimité, résume Laurence Schram. D’autres pays arabo-musulmans (Égypte, Palestine, Qatar, Arabie saoudite...) emboîtent le pas à l'Iran et soutiennent les négationnistes.

La dernière décennie connaît d'autres affaires, comme le procès, en 2007, de Faurisson contre Robert Badinter, qui l'a qualifié de «faussaire de l'histoire». Si le négationniste perd le procès, il gagne en médiatisation. En 2009, Dieudonné le fait monter sur la scène d'un de ses spectacles au Zénith sous les applaudissements du public. Aujourd'hui se pose la question de l'après-Faurisson. Vincent Reynouard, intégriste catholique et adepte de l'idéologie nazie, auteur de maintes thèses niant la Shoah, devrait lui succéder. Désormais, le point de rassemblement de tous ces mouvements négationnistes, c'est l'antisionisme radical.

\section{LE GÉNOCIDE ARMÉNIEN}

Au $19^{\mathrm{e}}$ siècle, les Arméniens, en tant que chrétiens, sont des citoyens de seconde zone dans un Empire ottoman musulman, explique Yves Ternon, docteur en histoire 
à Paris IV Sorbonne. Ils demandent des réformes au sultan, en vain. Très vite, ils sont perçus comme un ennemi intérieur, allié de la Russie. Les violences à l'égard des populations arméniennes débouchent sur le massacre de 300000 personnes en 1895. Mais ce qui mène au génocide, c'est l'apparition du parti Jeunes-Turcs et son arrivée au pouvoir en 1913. Au sein de ce parti, l’idéologie «la Turquie doit appartenir aux Turcs » domine. Et pour être Turc, il faut être musulman. Ce qui entraîne une épuration ethnique des populations chrétiennes sur les théâtres de combat avec massacre d'Arméniens, d’Assyriens et de chrétiens du Pont, précise Yves Ternon.

La destruction des Arméniens commence le 24 avril 1915 et la déportation est l'une des armes de ce génocide. Il fera près de 1,5 million de morts. Dès 1916, les premiers discours négationnistes justifient cette déportation. Ils invoquent, entre autres, le risque que les Arméniens ne collaborent avec l'ennemi russe. En 1923, avec l'apparition de l'État turc, les procès prouvent qu'il y a eu une organisation du génocide. Puis, sous l'État kémaliste, c'est le silence. Il persiste jusqu'en 1946, année où le terme «génocide » est évoqué au procès de Nuremberg. Les Arméniens s'en emparent lentement. Mais le vrai processus négationniste s'enclenche lors des 50 ans du génocide arménien, en 1965, lorsque le silence est brisé dans plusieurs pays. Depuis, à chaque fois qu'il y a une demande de reconnaissance du génocide d'une organisation, d'un État, les réactions sont les mêmes : allant d'une lettre d'ambassadeur, assortie de menaces, jusqu'au rappel du diplomate.

En 1992, lors de l'arrivée de l'AKP au pouvoir et du président Erdogan, l'AKP propose d'organiser une discussion entre Turcs et Arméniens. Sans résultat. Depuis plusieurs années, une partie de la société civile plaide pour la reconnaissance du génocide, en particulier à la suite de l'assassinat en 2007 du journaliste turcoarménien Hrant Dink par un nationaliste turc. Ce qui entraîne un durcissement du gouvernement Erdogan. Un constat également partagé par Dogan Özgüden, rédacteur en chef d'Info-Turk : l'opposition, les journalistes subissent des menaces, des académiciens sont dénoncés comme traîtres dans les journaux au service du pouvoir. Enfin, complète Yves Ternon, en 2015, pour contrer les commémorations du 24 avril en Arménie, le gouvernement turc a célébré, le même jour, le centenaire du débarquement allié dans les Dardanelles... qui a eu lieu le 25 avril !

\section{LE GÉNOCIDE TUTSI AU RWANDA}

Plus récent, le génocide tutsi au Rwanda constitue une autre problématique. D’emblée, Yves Ternon invite à être attentif aux choix des termes : «Il n’y a pas eu de génocide rwandais, mais un génocide tutsi au Rwanda », insiste-t-il. Et de remonter aux racines du génocide : quand la Belgique succède à l'Allemagne au Rwanda, les colons considèrent qu'il y a un peuple, les Hutus, et qu'un peuple colonisateur, les Tutsi, est arrivé. L'État belge fixe cette origine sur les cartes d'identité. En 1963, les Hutus prennent le pouvoir, chassent les Tutsi et les persécutent. Ceux-ci se réfugient 


\section{DOSSIER}

La pédagogie pour contrer le négationnisme (suite)

en Ouganda. Le FPR (Front patriotique rwandais) envisage un retour dans les années 1980. Dans les médias, les radios, dès 1993, on parle de la destruction des Tutsi. Le 6 avril 1994, après que l'avion présidentiel ait été abattu, c'est le déclic. Le génocide commence et dure jusqu’à l'arrivée en juillet du FPR. On dénombre entre 800000 et un million de morts.

Aujourd'hui, au Rwanda, les génocidaires et ceux qui ont participé ne peuvent pas nier le génocide. Les négationnistes se sont réfugiés ailleurs. Mais il existe d’autres attitudes négationnistes, pointe Yves Ternon: «Ce sont ceux qui se sentent coupables de ce génocide, mais ne veulent pas le reconnaître : à savoir, la Belgique, à l'origine de l'ethnicisation des populations, la France, avec l'opération Turquoise qui a servi à combattre le FPR et sans doute à armer des génocidaires, les USA, qui ont vu le risque de génocide, mais ne sont pas intervenus, et, enfin, l'ONU.» «Mais comme il n'est pas possible pour ces acteurs de reconnaître leur responsabilité, il faut alors expliquer, voire justifier le génocide», dénonce Yves Ternon. Il énumère les différentes théories mises en avant. Tout d'abord, la fureur populaire incontrôlable provoquée par l'explosion de l'avion. L'autre théorie qui remporte du succès est celle du double génocide : il y a eu plus de tués Hutus que Tutsi, surtout avec l'arrivée du FPR. «Dans les faits, il y a eu des crimes de guerre dans le Nord-Kivu, mais pas de génocide hutu au Rwanda », souligne-t-il. Enfin, il épingle Pierre Péan, qui vajusqu’à affirmer que le FPR a provoqué sciemment le génocide en abattant l'avion présidentiel... Une fois encore, les théories négationnistes sont assez proches des théories du complot.

\section{QUELS OUTILS POUR COMBATTRE LE NÉGATIONNISME ?}

Aujourd'hui, la loi permet de condamner les discours négationnistes. Éric David, président du Centre de droit international de l'ULB, en répertorie trois. Tout d'abord, il y a celles qui condamnent la négation des génocides perpétrés par les nazis (Allemagne, Autriche, Belgique). La deuxième catégorie de législations s'étend à tous les génocides et à tous les crimes de guerre.

_ Statue « En mémoire des victimes du génocide Rwanda 1994 » «Soit les autres législations qui condamnent ce qui a été fait par les nazis, mais aussi tout ce qui est repris à l'article 6 du jugement de Nuremberg (crimes contre la paix, crimes de guerre et crimes contre l'humanité)», explique Éric David. En Pologne, la législation vise les crimes nazis et les crimes soviétiques. En Espagne, elle concerne tout génocide, qui vise «à détruire tout ou partie d'un peuple en fonction de son appartenance ethnique, sociale, religieuse, politique, ou d'un handicap. » La législation suisse va plus loin, puisqu'elle concerne tous les crimes commis pour exprimer une haine raciale. Enfin, il y a des législations qui visent explicitement le négationnisme, si les crimes sont reconnus par des juridictions ou des autorités 
internationales (ex.: l'ONU qualifie le nettoyage ethnique de l'ex-Yougoslavie comme une «forme de génocide»). Enfin, il y a les législations qui condamnent toute tentative de nier ou minimiser "grossièrement» un génocide ou des crimes de guerre (Autriche, Belgique et Suisse). «Derrière ce déni, on retrouve la notion d'injure et donc de dommage, souligne Éric David, un terme où les juristes se retrouvent et pour lequel le Code civil suffirait pour condamner un négationniste.» Mais cet arsenal juridique est à double tranchant. Comme l'a montré Laurence Schram, un procès mené contre des négationnistes leur offre une visibilité médiatique encore plus grande. «Des lois existent, mais sont-elles utiles et efficaces ?» s'interroge-t-elle. «Les négationnistes les utilisent souvent pour se poser en victimes.»

Par ailleurs, ils désarçonnent très souvent leurs contradicteurs par leurs propos. Ils sont très attentifs au choix des termes et connaissent des détails très précis concernant la Shoah. D'où la deuxième arme, mise en avant par Laurence Schram : la pédagogie et l'enseignement. «Il faut faire de l'histoire et faire passer de manière pédagogique les résultats. Bien qu'on ait l'impression d'être saturé par la Shoah, quand on interroge les gens, on se rend compte qu'ils ne connaissent rien.» L'un des points positifs du négationnisme, selon elle, est «qu'il a poussé les historiens à faire des recherches et à vulgariser l'histoire.»

\section{LA PÉDAGOGIE À L'ÉPREUVE DU TERRAIN}

De manière générale, les enseignants et les intervenants dans les écoles perçoivent de plus en plus chez les élèves une certaine légitimité à dire ce qu'ils pensent. «Notre parole est sans cesse remise en question sur le plan historique ou de l'actualité, pointe une enseignante. Les gens adhèrent facilement aux théories du complot.» Un autre observe : «Dans les classes, concernant la Shoah, on a des réflexions qui sont souvent assez frontales : "Vous exagérez", "Une haine pareille, ce n'est pas possible, il n'y a pas de fumée sans feu”. » D’autres sont surpris par la capacité à argumenter de certains élèves.

Mais il n’y a pas que le génocide juif qui est visé. Au cours des débats, les participants ont pu observer à quel point reste sensible la question du génocide tutsi au Rwanda. Certains plaident pour la reconnaissance des victimes hutues modérées. Tandis que d'autres, comme les représentants de l'asbl Ibuka Mémoire et Justice ${ }^{7}$, s'insurgent contre une tendance à vouloir minimiser le génocide tutsi en mettant en avant les victimes hutues. Sur le terrain, certains vont plus loin : le Centre communautaire laïc juif $(\mathrm{CCLJ})^{8}$ a parfois été confronté à des partisans du double génocide au Rwanda. Mais c'est la question arménienne qui provoque le plus de débats houleux, voire violents. Dans le cadre d'une exposition consacrée au génocide arménien, Philippe Marchal, des Territoires de la Mémoire, rapporte s'être retrouvé face à une fille de 12 ans niant la vérité historique. Il y a aussi eu quelquefois certaines recommandations de ne pas trop parler de la Turquie et du génocide arménien. Dans l'ensemble, il s'agit surtout de remises en question... « Il y a parfois eu des visites de
(7) Asbl regroupant les survivants du génocide perpétré contre les Tutsi au Rwanda en 1994 http:// ibuka.be/

(8) http://www.cclj.be 
La pédagogie pour contrer le négationnisme (suite) courtoisie qui dans certains cas ont tourné à l'intimidation par rapport au génocide arménien », précise Ina Van Looy du CCLJ. Selon elle, le génocide arménien provoque le plus de violences chez les élèves qu'ils rencontrent. La première réaction est : «Mais M'dame, on n'a pas fait ça. » «Oui, eux, les élèves ne l'ont pas fait. Mais on doit les sortir de cette éducation négationniste. Il faut apprendre à les démonter pour y arriver. On a besoin des juristes pour qualifier les crimes, insiste Ina Van Looy. On doit être extrêmement prudents et attentifs aux mots que l'on utilise. Parce que les négationnistes et les génocidaires y sont très attentifs. C'est pour cela qu'aujourd'hui, on parle du génocide des Arméniens, des Assyriens et des chrétiens du Pont.» Une enseignante en histoire a expliqué que le problème n'était pas toujours les élèves, mais certains professeurs de religion qui refusent de visiter des expositions sur le génocide arménien et excluent de facto leurs élèves de ces activités.

\section{CONCURRENCE VICTIMAIRE}

Un autre discours - qui peut être apparenté à une minimisation d'un génocide porte sur la concurrence victimaire. Le conflit israélo-palestinien est souvent brandi lorsqu'est évoquée la Shoah. Et la comparaison oblige à répondre. Lorsqu'Ina Van Looy, du CCLJ, s'entend dire «Hé M'dame, et le génocide des Palestiniens ?» Elle en discute avec les élèves : «Oui, il y a une guerre. Non, il n’y a pas de génocide.» Un enseignant du secondaire observe que les élèves réagissent de manière différente. C'est le cas des élèves polonais, qui ont le sentiment de se faire confisquer leur histoire, lorsque l'on évoque le génocide juif.

Le génocide tutsi au Rwanda reste un objet de concurrence victimaire, entre autres via les partisans de la thèse du double génocide. «Souvent, on entend, "il y a eu un génocide, mais...”, s'emporte un représentant de l'asbl Ibuka. Pourquoi il y a toujours des "mais" ? Quand on commence à dire "mais", pour moi, c'est de la minimisation, c'est déjà une forme de négationnisme. Il ne faut pas comparer ce qui n'est pas comparable.»

Pour Laurence Schram, la comparaison est nécessaire : «Quand il y a une procédure d'extermination, les génocidaires ferment les frontières pour éviter que les gens ne fuient. C'est le cas dans le cadre du génocide arménien, juif, tutsi. Il y a donc une différence entre les génocides et le nettoyage ethnique, où l'on fait fuir des populations - comme en ex-Yougoslavie. Je ne minimise pas la souffrance des populations, c'est le processus que je mets en question. La mise en concurrence est une stratégie négationniste.»

\section{LA NÉBULEUSE DU WEB}

Aujourd'hui, l'école n'est plus le principal lieu d'apprentissage des adolescents. Le Web regorge d'informations - pas toujours fiables - utilisées autant par les adultes que les élèves. Frédéric Crahay, directeur de la Fondation Auschwitz - Mémoire d'Auschwitz ASBL constate qu'il est très facile d'arriver sur des sites négationnistes, 
qui ne se présentent pas comme tels. «Il y a, par exemple, beaucoup d'écrits sur la technicité des chambres à gaz», note-t-il. Il évoque la fois, où interpellé par une mère, il a été amené à discuter avec ses deux fils qui connaissaient une quantité d'aspects techniques qu'ils avaient découverts sur des sites négationnistes. Pour les amener à s'interroger sur la fiabilité de leurs sources, il leur a présenté des documents plus difficiles à trouver sur le Net, tel le télégramme du 11 janvier 1943 du SS Höffle à Adolf Eichmann, renseignant le nombre de victimes dans les centres d'extermination de Majdanek, Sobibór, Treblinka et Bełżec.

«Le problème, note Johan Puttemans, responsable pédagogique à

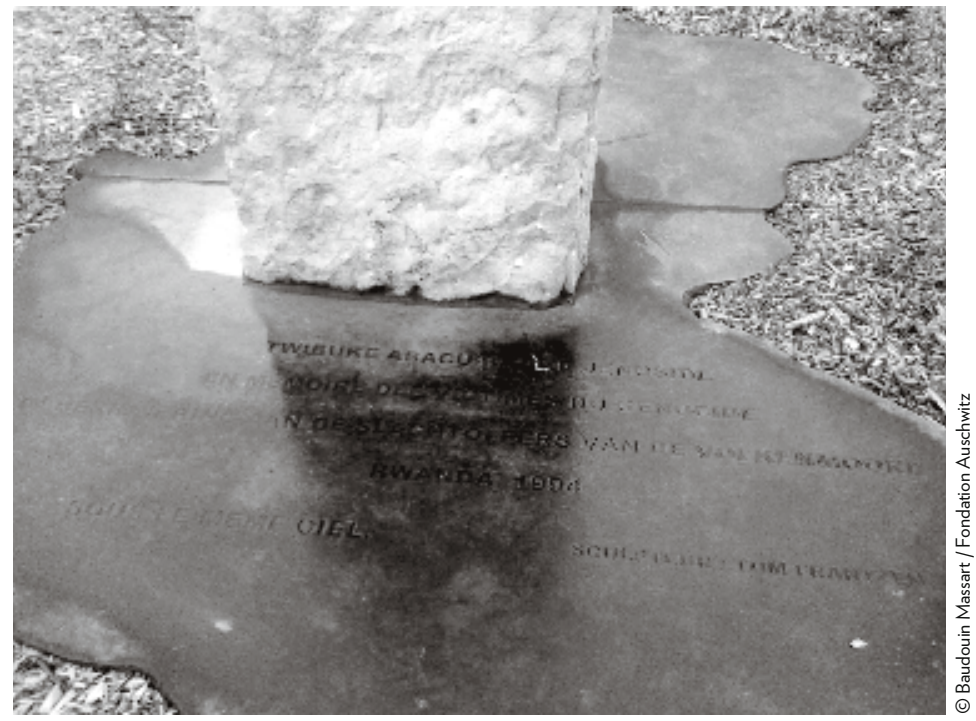
la Fondation Auschwitz - Mémoire d'Auschwitz ASBL est que pour beaucoup de jeunes, comme pour beaucoup de monde, quand c'est sur Internet, c'est la vérité.»

\section{QUAND LA SHOAH LASSE}

Mais la lassitude peut l'emporter aussi sur le négationnisme. Face au «Encore Auschwitz! » spontané des élèves, les pédagogues doivent s’adapter et faire preuve de créativité pour remobiliser les élèves. Ces derniers se sentent déprimés ou en colère face à de tels événements : «Pourquoi ils n’ont pas arrêtéles trains? Pourquoi ils n'ont rien fait? Qu'est-ce qu'on peut y faire ? Ne nous en parlez plus, cela ne sert à rien.»

Pour changer d'angle d'attaque, un professeur d'histoire insiste sur la nécessité de rappeler à l'élève qu'il y a toujours une démarche scientifique derrière les faits, que «l'histoire que l'on raconte est basée sur des choses réelles.»

Élias Constas, enseignant et collaborateur scientifique aux Musées royaux d'Art et d'Histoire, prône l'éveil à la critique des élèves : «On a souvent tendance à infantiliser les élèves. » Il propose un exercice sur le thème des caricatures, où ils présentent des sketches de Dieudonné et des caricatures de Charlie Hebdo. En regard de chaque sujet, il pose des questions fermées avec des références légales : ce sketch/cette caricature fait-il/elle rire? Fait-il/elle la défense des crimes de guerre, des crimes contre l'humanité ? Incite-t-il/elle à des actes terroristes ? Tend-il à la discrimination, la haine, la violence envers des personnes? etc. En fonction de ces «oui » et ces «non », l'élève prend conscience par lui-même de ce qui est autorisé ou pas. Il suggère aussi d'utiliser les pavés de mémoire comme parcours pédagogique qui favorise les questions-réponses. 


\section{DOSSIER}

La pédagogie pour contrer le négationnisme (suite)
_ Les «pavés de mémoire», un outil pédagogique pour aborder la Shoah au niveau local. Ici, au 59 boulevard du Midi, à Bruxelles.
(9) Cela s'observe jusque dans la proposition de résolution relative à la commémoration du centenaire du génocide arménien, adoptée à la Chambre le 23 juillet 2015.

\section{CONCLUSION}

Lutter contre les négationnistes nécessite d'être bien armé. Il faut donner des réponses et poser les bonnes questions. Des questions qui ne manquent pas pour Johan Puttemans : «Pourquoi le centre d'extermination de Birkenau a-t-il passé commande de détecteurs de Zyklon B, si on ne gaze pas, et en plus pour un bâtiment dénommé «morgue»?»; «Pourquoi passer commande d'une porte étanche au gaz avec judas pour une morgue?», etc. Autant d'interrogations qui mettent à mal les discours négationnistes. Elles sont aussi applicables aux publics jeunes. Plusieurs intervenants affirment qu'il ne faut pas chercher à convaincre les jeunes, mais les amener à s'interroger, une démarche indispensable pour développer leur esprit critique.

Encore faut-il que les États adoptent une position claire par rapport aux génocides et à leurs passés respectifs. Il est difficile pour un État de faire la leçon à un autre, s’il ne reconnaît pas ses propres torts. La Belgique a tardé à reconnaître sa responsabilité dans la Shoah. Au Rwanda, le système colonial de classification ethnique a favorisé le génocide des Tutsi. Enfin, l'État belge reste frileux par rapport au génocide arménien ${ }^{9}$, certains partis redoutant de s'aliéner leurs élus d'origine turque et de perdre leur électorat. De quoi laisser le champ libre aux négationnistes.

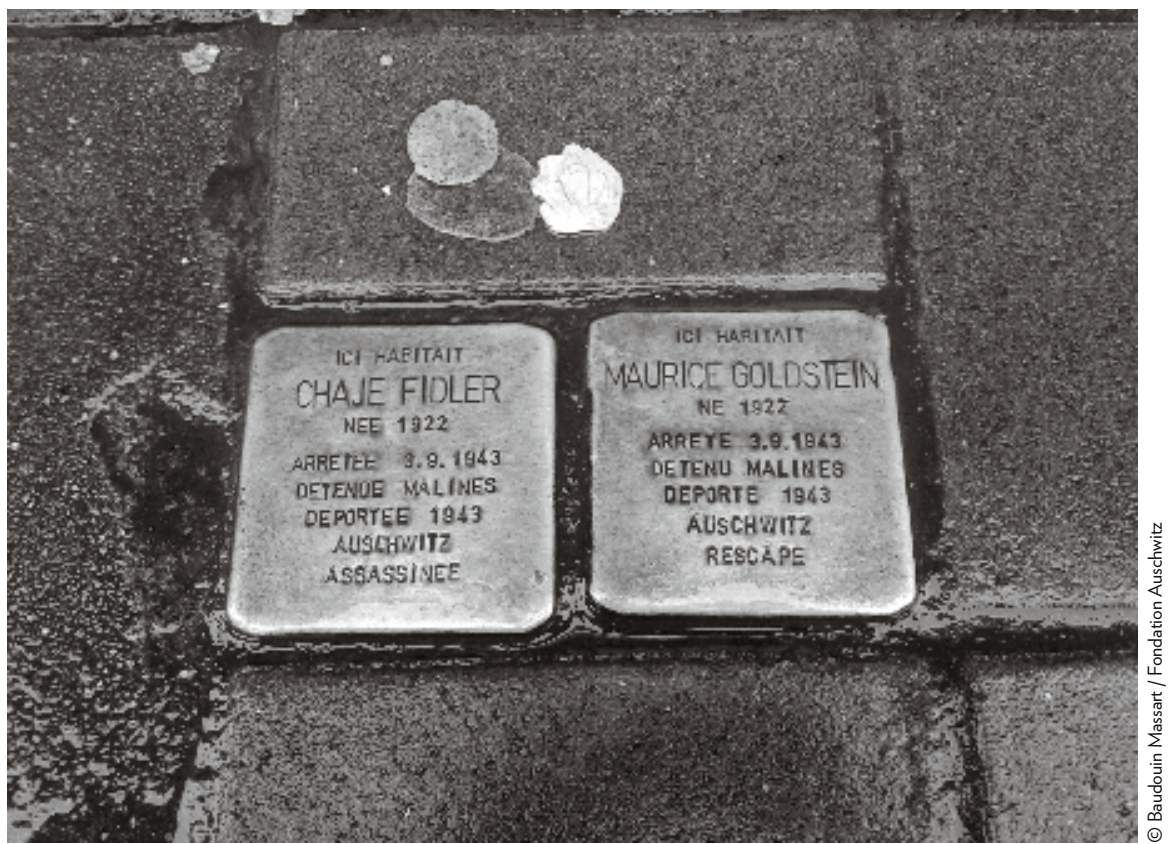

